\title{
Developing an \\ Integrated \\ Location and \\ Information \\ Database for \\ Teaching Plant \\ Identification \\ and Use
}

\author{
Cathy Sabota, Caula A. Beyl, \\ and Gokul Ghale
}

Additional index words. landscape design, cultivar, database manager, computer, graphics

Summary. The landscape plants that exist on the Alabama A\&M University, Normal, campus are readily accessible for a plant identification and use course. Managing location, health, and cultivar information is critical to optimizing this resource. As a classroom assignment, campus plants were inventoried; entered into FileMaker Pro 2.1, a relational database manager; characterized; and assigned locations on campus. The campus map was scanned using a Microtek Scanmaker IIxe and the image was imported into MacDraw II. A symbol library, which included symbols for trees, shrubs, and groundcovers, was developed by scanning hand-drawn images and then importing them into MacPaint. These bit-mapped images were duplicated as often as necessary and placed in appropriate locations on the campus map in MacDraw II. Students were exposed to landscape plant materials, database managers, and computer graphics capabilities. This approach has other advantages: database information can be easily coordinated with physical location, plants can be sorted based on their characteristics, and information can be routinely and easily revised and updated. The database is used in the landscape plant materials class as a teaching tool and for self-guided tours.

\footnotetext{
Associate professor, professor, and graduate research as sistant, respectively; Department of Plant and Soil Scicnce, Alabama A广M University, Normal, AL 35762.

The cost of publishing this paper was defrayed in part by the payment of page charges. Under postal regulations, this paper therefore must be berely marked advertisement solely to indicate this fact.
} 
$\mathrm{T}$ caching identification and use of landscape plants always includes learning plant characteristics and, optimally, involves viewing the plants in their landscape setting. For most plant identification courses, this requires a map of available resources containing plant locations and detailed plant descriptions that can be distributed to students or used to" plan field trips to visit the selected plants. Although campus plants are often readily available for teaching needs, most campus sites have constantly changing landscapes, with plants continually being added or removed. Hand--drawn maps would have to be redrawn every time the information needed to be updated. To accommodate these changes easily, a computer database of plants is essential. In addition to locating and identifying plants, descriptions of all significant plant characteristics (descriptors), including bud type, foliage colors, texture, size, moisture preferences, soil tolerances, habit, flowering season, color, bark features, and shade tolerance, can be logged. Once the information is entered into the database, an instructor or student can sort on a specific descriptor and obtain all plants that meet that criterion. Such a database is a useful tool for teaching landscape design or specialized topics such as shade tolerance, flowering sequences, and use of color.

In addition to classroom use, a combined approach such as this is valuable to extension horticulture. Extension home horticulturists are often asked by clientele about plants for shade

Fig. 1. The typical output report for Betula platyphylla japonica, which includes all the input characteristics used in the Alabama A.rM Univ. campus plants database. tolerance, small evergreens for foundations, attracting birds, or specific flower colors. Software can sort on various descriptors, give locations where the plants can be seen, and teach homeowners how to identify and use these plants. The database can be customized for regional preferences and availability of plants.

The database described in this paper was developed as a student project. The technique was adapted for classroom use. The use of this material in the classroom was intended to help students gain computer expe- rience in dealing with and understanding relational databases and to become more familiar with plant classifications and characteristics. We have developed a tool that allows us to exploit the campus plants in a customized, interactive way to allow selfguided tours and to store information about campus plants that can be easily updated.

\section{Hardware and software requirements}

Probably the most time-consuming aspect of this approach to teaching

\section{Betula platyphylla japonica}

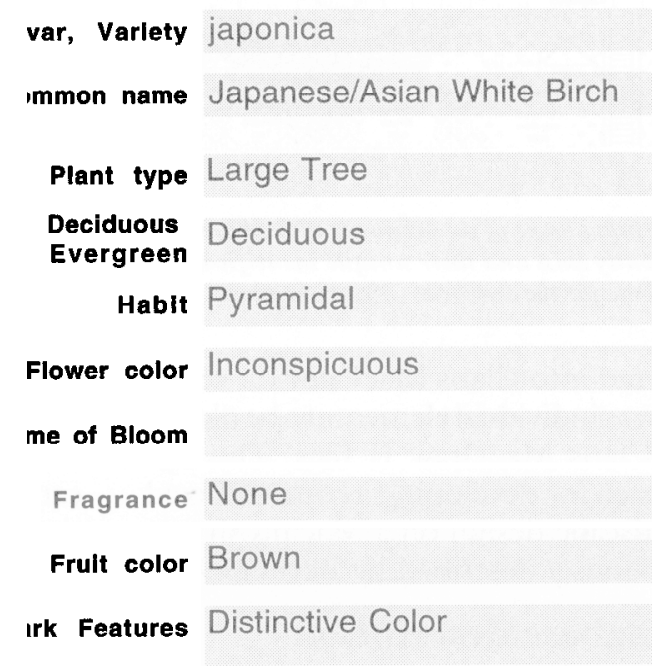

Leaf Shape
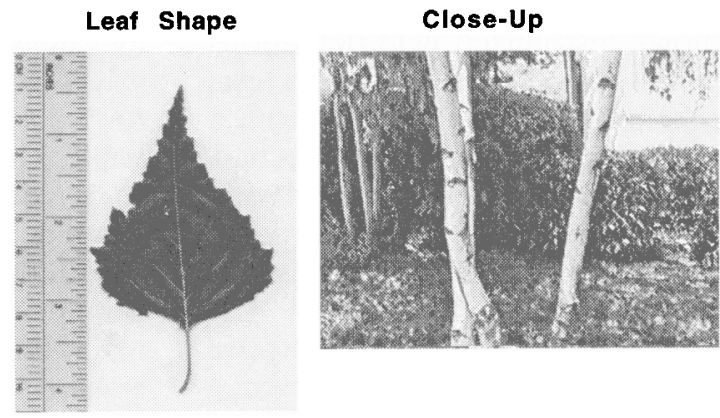

Environmental Requirements

Full Sun Tolerance Full

Shade Tolerance Partial

Water Usage Medium

Drought Tolerance Partially Drought Tolerant

Flood Tolerance Poor Flood Tolerance

Pollution Tolerance Unknown

Lowest Hardiness Zone

Identification Number
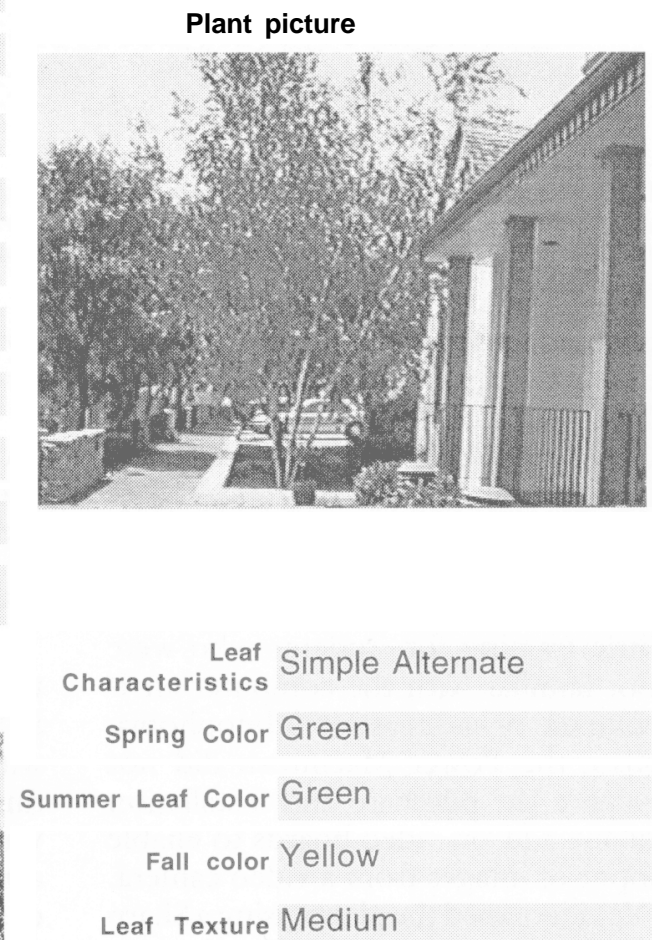

Landscape Value Specimen or lawn tree

Location Hillcrest

Condition Healthy, good specimen 


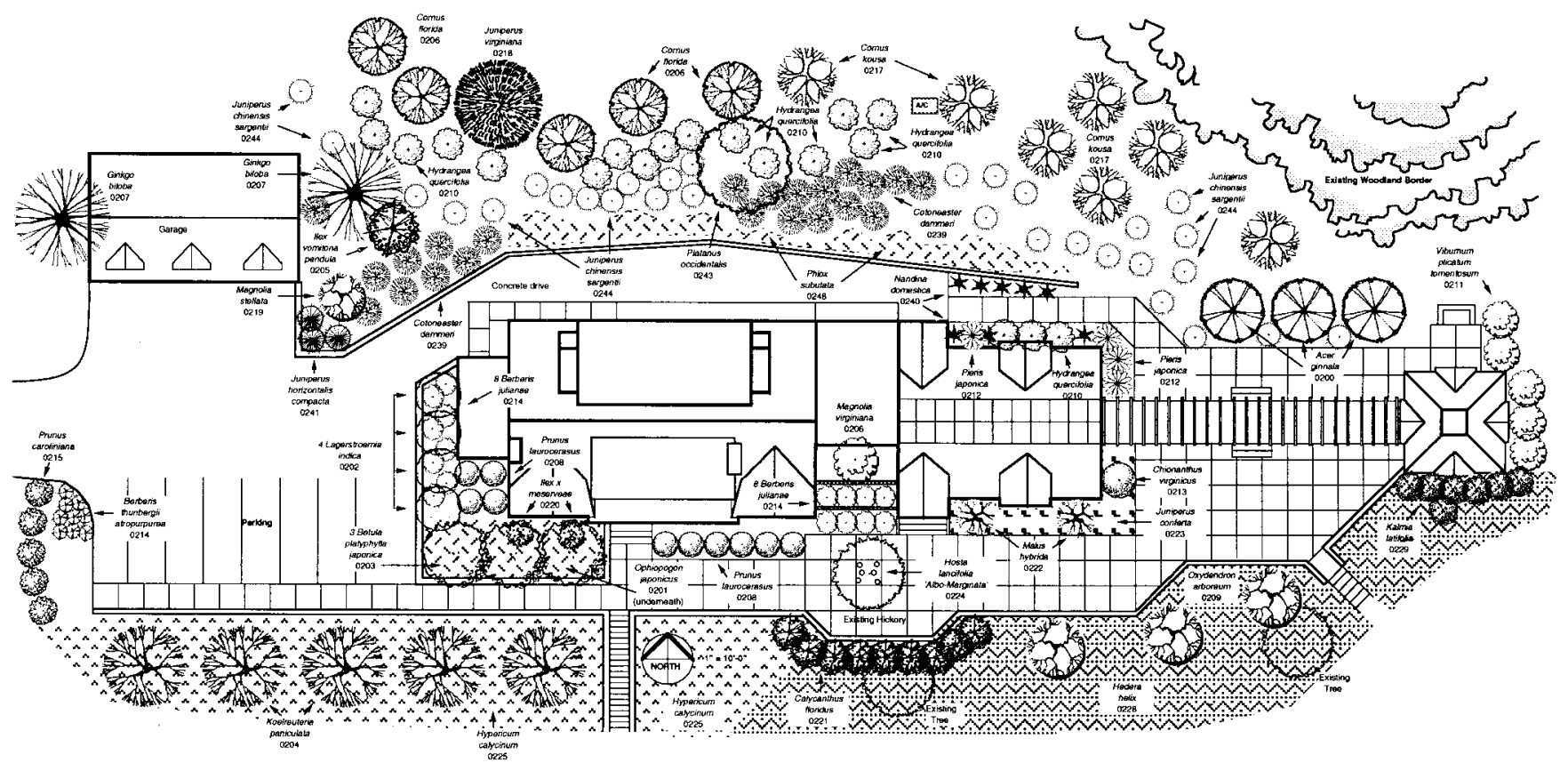

Fig. 2. The landscape map of Hillcrest, the traditional campus home of the president of Alabama Aひ M Univ., illustrates the plant materials as they currently exist and their unique identification numbers. These can then be used to refer back to the database for the characteristics of the plant material.

plant identification and use was the collection and input of data into the database. The database software we used was Claris FileMaker Pro 2.1 installed on a Power Macintosh 7 100/ $66 \mathrm{AV}$ with $24 \mathrm{MB}$ of RAM. This configuration was selected for two reasons .It is user-friendly and required little training for students who were not familiar with the computer environment or had a case of technophobia. Also, the Power Macintosh did not require the purchase and installation of any add-on video boards to enable input of images from a video camera. We videotaped the plants using a Sharp VHS video camera, and then the best views were frame-captured using Video Monitor 1.0.1, down-loaded to the computer, and transferred to Adobe Photoshop 2.5 for cropping or enhancement. Individual leaves and fruit were scanned using a Microtek Scanmaker IIxe color flatbed scanner and then saved as Adobe Photoshop 2.5 files. The database files included color and black-and-white images that had been transferred from Adobe Photoshop 2.5 files and were printed on a LaserWriter Pro [600 dots per inch (dpi)] (Fig. 1 ) or a Color StyleWriter Pro printer. Maps were created using Claris MacDraw 11 with landscape symbols for trees and shrubs that had been hand-drawn, scanned, and saved as PICT images and im- ported into Claris MacPaint for selection as individual elements to be placed in Claris MacDraw II files. This approach for developing a computerized landscape design on a Macintosh was previously described by Beyl ( 1990).

\section{Database description}

FileMaker Pro software was used to develop the plant database, because it allows customized field definitions with the flexibility of importing text, numbers, dates, and pictures from a variety of sources. Fields are plant characteristics that were used to classify descriptor values of a plant. A typical field was flower color, and the descriptor values within it included blue, yellow, or red. FileMaker Pro software allowed us to predefine these descriptor values, reduced data entry time, and ensured some degree of standardization. The sort option enabled selection of all entries in the database that matched certain specified criteria, such as a search for all species on campus with blue flowers, or all species on campus that are pollution-tolerant. The software also provided for various layouts that were custom-designed, enabling output of only user-selected field information in report format. This was useful for the student who wanted to printout only shade-tolerant plants, their names, and locations, for example.

\section{Database development}

Characteristics used for identification and use that were included in the FileMaker Pro spreadsheet could be open-ended or predefine with a value list. Open-ended fields included the genus, species, common name, cultivar or variety, and a comments section. The remaining fields were easily categorized with a value list. The value list was a set of options selected for a particular characteristic, such as habit. The options for habit included pyramidal, vining, rounded, vaseshaped, multi-stemmed, broadly rounded, weeping, broad pyramidal, upright, columnar, spreading, and formal. These options were selected from a pull-down menu when entering data on each landscape species.

The information for each plant species was obtained from personal experience and various plant authorities, including Bridwell (1994), Dirr (1983), Flint (1983), and Halfacre and Shawcroft (1989). Each plant or plant grouping was located on a campus map and cross-referenced to the database with a unique identification number. A campus map was developed to record the locations of all of the plants in MacDraw II using a technique previously detailed by Beyl (1990). The symbol library, which included symbols for trees, shrubs, and 
groundcovers, was developed by scanning hand-drawn images and importing them into MacPaint. Individual bit-mapped images could then be selected, duplicated as needed, and placed inappropriate locations on the campus map in MacDraw II (Fig. 2).

\section{Obtaining and importing pictorial images into the database}

Individual leaves or fruit were taken from each plant and placed on the Microtek Scanmaker IIxe color flatbed scanner next to a ruler for scale. Images scanned in color on the scanner required up to $3.2 \mathrm{MG}$ of memory to store and up to an hour to select and transfer the image to the database file. As an alternative, this procedure was modified to include video image closeups of the leaves, stems, and fruit because the video images required only $65 \mathrm{~kb}$ of memory and very little time to transfer files. The appearance of the plant on the site was videotaped with a Sharp video camera to enable the habit and form to be shown. Images captured on videotape could be downloaded to the Power Macintosh via an audio-video input cable using the application Video Monitor 1.0.1, allowing a single frame to be selected. Then they were transferred to Adobe Photoshop 2.5 so that any portion of the image could be chosen, reduced or enlarged, and saved at 100 to $600 \mathrm{dpi}$ in color or black and white (Fig. 3).

As an alternative to capturing your own images, more than 3500 photographs of more than 1800 plant species are available on CD-ROM from the Univ. of Florida (Gilman, 1994). This is an excellent resource if you are using DOS and Windows applications. The software also contains line drawings and more than 3000 pages of text with extensive morphological characteristics and plant use suggestions. From this software, lists can be created to match specific characteristics, desirable ornamental attributes, or both. Students can also use this database to identify an unknown plant specimen. Other features of the program allow

Fig. 3. An image as it appears when it is first frame-captured from the video camera into an Adobe Photoshop 2.5 file. The image depicted was used to capture bark detail of Betula platyphylla japonica. viewing insect and disease problems and access to up-to-date control recommendations.

\section{Problems with FileMaker Pro database}

The problems associated with using the FileMaker Pro database with picture images are not numerous or insurmountable. As mentioned earlier, images that were scanned in color and stored at high resolutions (>100 dpi) consumed up to 3.2 MBs per image. The use of imported video rather than scanned images reduced the amount of storage and memory required, but the picture quality on the monitor and print quality was reduced.

Developing a library of imported color images or high resolution images for the landscape plants database requires a great deal of memory. A dedicated hard drive or peripheral storage device such as a SyQuest drive is probably the best approach for storing the files.

Printing files that contain many images can take along time. The axiom "the more RAM, the better" was certainly true here. This work was done on a computer with $24 \mathrm{MB}$ of RAM, and some of the color files took from $30 \mathrm{~min}$ to $8 \mathrm{~h}$ to print.

One other minor limitation is that the sort option was limited to predefine value lists only, since openended values would not contain predefine sortable values. This means that optimally as much of the database as possible should be in the form of predefined value lists to maximize the utility of the sort feature.

\section{Student response to its use}

The program, initiated as a student special project, was later adapted for use as classroom teaching notes, self-guided tours for students, and to train grounds-maintenance personnel.

Students were excited about using the computer as an approach to learning about landscape plants and their uses. The classification by characteristics sparked debate in class discussions, because the students would contrast information from different sources. Students also liked the pictorial representations of the plant at the actual site, because it reinforced their impression of the plants in situ that they first observed when taking the tours.

Lindsey et al. (1994) evaluated the value of computer-based learning of landscape plant materials and their uses. Features of his program included a slide show that allowed students to select which plants and characteristics to be viewed and in what order with the option of an interactive quiz. The system is being used to determine how students learn the information presented to them using computer technology and which features are most useful in improving skills and knowledge. Computer-based learning as a supplement to regular classroom instruction and outdoor labs did not

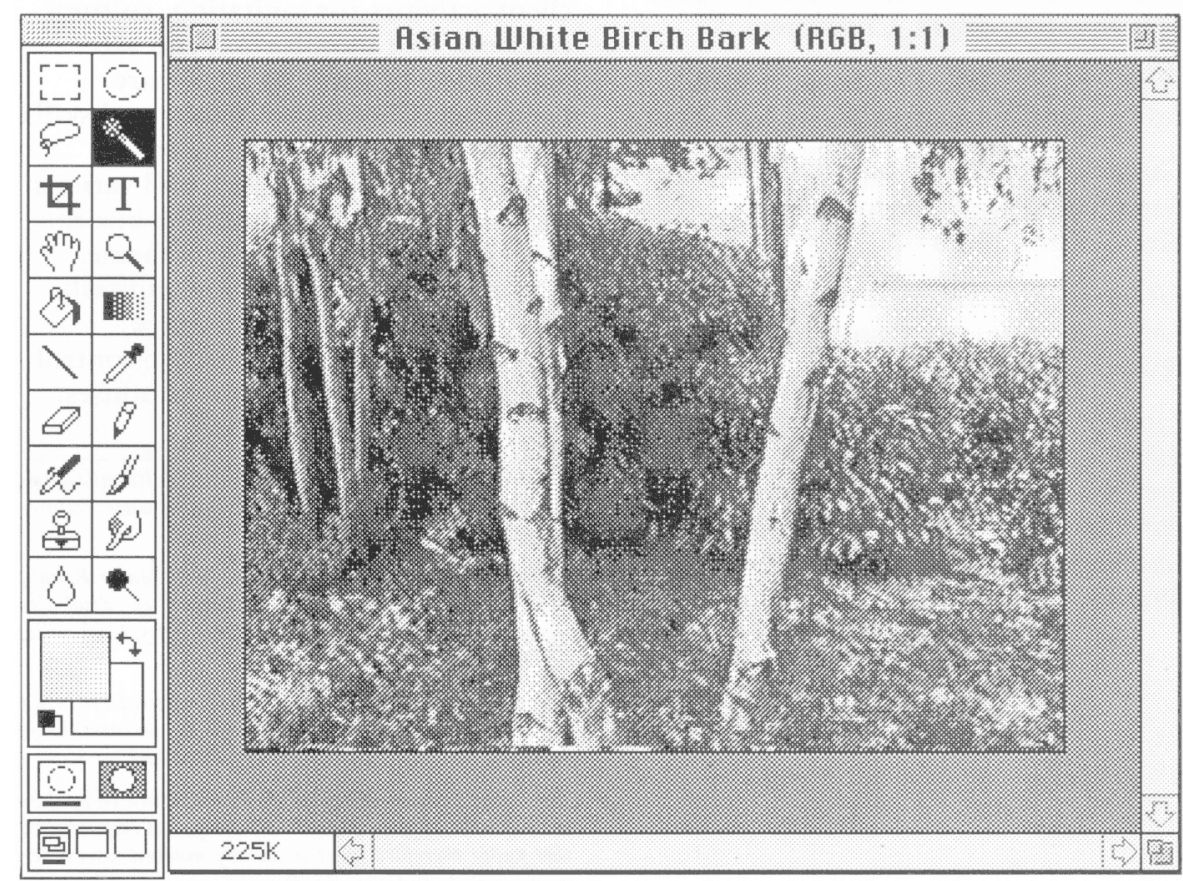




\section{TEACHING METHODS}

improve student grades. However, in another study, students who were able to review pictures on the computer performed significantly better on that part of the exam (Kling, 1995, personal communication).

\section{Other potential uses for this approach}

A complete database is a valuable resource for landscape and horticultural instructors, extension agents, landscape professionals, nurseries and garden centers, and homeowners. Once the files are created for a particular region, they can be packaged with instructions for their use and modification enabling addition or deletion of plants. This same approach is not limited to woody ornamental but could be adapted for perennials, annuals, and house plants. Students using this package are exposed to landscape plants and their characteristics and the use of a database manager to organize and sort information and computer graphics capabilities.

\section{Literature Cited}

Beyl, C. 1990. Low cost, computer-aided landscape design using the Macintosh computer. HortScience 25:353-356.

Bridwell, F.M. 1994. Landscape plants. Their identification, culture, and use. Delmar, Albany, N.Y.

Dirr, M.A. 1983. Manual of woody landscape plants: Their identification, ornamental characteristics, culture, propagation and uses. Stipes, Champaign, 111.

Flint, H.L. 1983. Landscape plants for eastern North America. Wiley, New York.

Gilman, E.F. 1994. Using CD-ROM computer technology to teach plant materials. HortScience 29:461-2.

Halfacre, R.G. and A.R. Shawcraft. 1989. Landscape plants of the southeast. Sparks, Raleigh, N.C.

Lindsey, C., G. Klivg, and M. Zampardo. 1994. Software usage patterns and student preferences in the evaluation of a computer-based interactive program for woody landscape plant instruction. HortScience 29:488. 